existing models noted: a shift of attention to a specialist, but not to a client; borrowing static and absolutistic ideas about cultures, without consideration of cultures development and interaction.

Conclusions: There is a trend in contemporary global world for broad research and development of cultural competence that improve professional qualities of healthcare professionals and provide psychological assistance to representatives of different ethnic and culture groups, confessions and minorities. The reported study was funded by the Russian Foundation for Basic Research, project number 17-29-02506.

Disclosure: No significant relationships.

Keywords: mental health; cultural competence; global world

\section{Depressive disorders}

\section{8}

\section{Adolescent oral contraceptive use and future major depressive disorder}

\author{
A. De Wit ${ }^{1 \star}$, C. Anderl ${ }^{2}$, E. Giltay ${ }^{3}$, T. Oldehinkel ${ }^{1}$ and F. Chen ${ }^{2}$ \\ ${ }^{1}$ Psychiatry, University Medical Center Groningen/University of \\ Groningen, Groningen, Netherlands; ${ }^{2}$ Department Of Psychology, \\ University of British Columbia, Vancouver, Canada and ${ }^{3}$ Department \\ Of Psychiatry, Leiden University Medical Center, Leiden, Netherlands \\ ${ }^{*}$ Corresponding author. \\ doi: 10.1192/j.eurpsy.2021.306
}

Introduction: Previously reported associations between oral contraceptives (OCs) use and depression have been conflicting. Insight into the impact of analytical choices on the association may help to reconcile previous heterogeneous findings.

Objectives: We aimed to examine the association between adolescent OC use and subsequent depression risk in early adulthood analyzing all theoretically justifiable models.

Methods: Women from the prospective cohort study Tracking Adolescents' Individual Lives Survey (TRAILS) were included in this study. All justifiable associations between adolescent OC use (ages 16-19 years) and major depressive disorder (MDD) in early adulthood (ages 20-25 years) as assessed by the Diagnostic and Statistical Manual of Mental Disorders-IV oriented Lifetime Depression Assessment Self-Report and the Composite International Diagnostic Interview were tested.

Results: A total of 818 analytical models were analyzed in 534 adolescent OC users and 191 nonusers. Overall, there was a tentative association of adolescent OC use and an episode of MDD in early adulthood (median odds ratio $[\mathrm{OR}]_{\text {median }}=1.41 ; \mathrm{OR}_{\min }=1.08$; $\mathrm{OR}_{\max }=2.18$, permutation testing $\mathrm{p}$-value $1=.052$, and $\mathrm{p}$-value $2=$ .046 ), which was primarily driven by the group of young women with no history of $\mathrm{MDD}\left(\mathrm{OR}_{\text {median }}=1.72 ; \mathrm{OR}_{\min }=1.21 ; \mathrm{OR}_{\max }=2.18\right.$, both permutation testing $\mathrm{p}$-values $=.02$ ).

Conclusions: Adolescent OC use was associated with an increased risk for experiencing an episode of MDD, but only among women with no history of MDD in adolescence. Understanding the potential side effects of OCs will help women and their doctors make informed choices when deciding among possible methods of birth control.

Disclosure: No significant relationships.

Keywords: Oral contraceptives; adolescence; Depression

\section{9}

Exploring predictors of depressive symptoms in patients with multiple sclerosis: The effect of neuropathic pain, shame, and mindfulness

T. Carvalho ${ }^{1,2}$, L. Benedito $^{1}$ and C. Gomes ${ }^{3 *}$

${ }^{1}$ Psychology, Instituto Superior Miguel Torga, Coimbra, Portugal;

${ }^{2}$ Faculty Of Psychology And Educational Sciences, University Of Coimbra, Center for Research in Neuropsychology and CognitiveBehavioral Intervention (CINEICC), Coimbra, Portugal and ${ }^{3}$ Clínica de Saúde Psiquiátrica de Coimbra - Casa da Oliveira, Coimbra, Portugal

${ }^{*}$ Corresponding author.

doi: 10.1192/j.eurpsy.2021.307

Introduction: Multiple Sclerosis (MS) is a chronic inflammatory, immune-mediated, demyelinating disease of the central nervous system, with a progressive course. It is potentially disabling and affects mainly young adults. Depression is the mental disorder with the greatest comorbidity with MS and tends to worsen its symptomatology and course. However, knowledge about the predictors of depression in patients with MS is scarce.

Objectives: This preliminary study aimed to verify whether neuropathic pain (NP), internal (IS) and external (ES) shame and mindfulness predict depressive symptoms in patients with MS.

Methods: This cross-sectional study included a convenience sample of 95 patients diagnosed with MS and without other identified neurological diseases. Participants completed the Depression Subscale of the Depression, Anxiety and Stress Scales-21, the Analogue Pain Scale of the Pain Detect Questionnaire, the External and Internal Shame Scale, and the Mindfulness Subscale of the Self-Compassion Scale.

Results: All potential predictors exhibited significant correlations with depressive symptoms and significantly predicted this symptomatology in simple linear regression models. Thus, they were included as covariates in the multiple linear regression model. This model explained a high percentage of the variance of depressive symptoms (40.5\%) and identified NP, IS and mindfulness as significant predictors.

Conclusions: Interventions aimed at preventing/reducing depression in patients with MS should minimize IS and develop mindfulness and NP coping skills, in order to promote mental health in this target population and possibly prevent the exacerbation and progression of MS symptomatology.

Disclosure: No significant relationships.

Keywords: Multiple sclerosis; depressive symptoms; predictive model

\section{1}

Baseline EEG-correlates of responders/non-responders to combined antidepressive treatment including transcranial magnetic stimulation

\section{E. Iznak ${ }^{1 *}$, A. Iznak ${ }^{1}$ and I. Oleichik ${ }^{2}$}

${ }^{1}$ Laboratory Of Neurophysiology, Mental Health Research Centre, Moscow, Russian Federation and ${ }^{2}$ Clinical Department Of Endogenous Mental Disorders And Affective States, Mental Health Research Centre, Moscow, Russian Federation

${ }^{*}$ Corresponding author.

doi: 10.1192/j.eurpsy.2021.308 
Introduction: Use of combined antidepressive treatment included high-frequency rhythmic transcranial magnetic stimulation (rTMS) of the left dorsolateral prefrontal cortex (DLPFC) is one of the ways for overcoming of pharmaco-resistance in depressive patients.

Objectives: The aim of the study was the search for possible EEG predictors of antidepressive effects of rTMS of the left DLPFC in combined treatment of depression.

Methods: 30 female in-patients (F31.3, F33.0, F33.1, by ICD-10; 20-50 years, mean age $36.9 \pm 10.3$ ) with pharmaco-resistant depression were enrolled in the study. Treatment included antidepressants (mainly SSRI) and a 3 -week course of rTMS $(20 \mathrm{~Hz})$ of the left DLPFC. Correlations between pre-treatment EEG spectral power values, and post-treatment quantitative clinical assessments of patients were analyzed. Responders/non-responders were determined by standard criteria of $50 \%$ decrease in HDRS- 17 scale total scores after treatment course.

Results: Responders (23 out of 30$)$ revealed significant $(\mathrm{p}<0.05)$ negative correlations between post-treatment HDRS-17 scores and pre-treatment EEG spectral power in theta-2 $(6-8 \mathrm{~Hz})$ and alpha-1 $(8-9 \mathrm{~Hz})$ frequency sub-bands in the parietal-occipital-posterior temporal leads. Non-responders (7 out of 30) showed negative correlations between the post-treatment HDRS-17 scores and pre-treatment theta-2 EEG spectral power in the frontal-centraltemporal regions of the right hemisphere.

Conclusions: Even brief course of rTMS of the left DLPFC enhances the action of antidepressants, and allows overcoming partially the pharmaco-resistance in depressive patients. Baseline values of theta-2 and alpha-1 EEG spectral power may serve as possible predictors of the effects of combined antidepressive therapy including rTMS. The study supported by RBRF grant No.1801-00029a.

Disclosure: No significant relationships.

Keywords: transcranial magnetic stimulation; baseline EEG; prediction of treatment effects; pharmaco-resistant depression

\section{2}

Individual dynamics of daily life functioning of reward system can predict future level of depressive symptoms

A. Kuranova ${ }^{1 \star}$, A. Martinez ${ }^{2}$, M. Wichers ${ }^{1}$, J. Wigman ${ }^{1}$ and S. Booij ${ }^{1}$

${ }^{1}$ Department Of Psychiatry, Interdisciplinary Center Psychopathology And Emotion Regulation (icpe), University of Groningen, University Medical Center Groningen, Groningen, Netherlands and ${ }^{2}$ Faculty Of Psychology And Neuroscience, Maastricht University, Maastricht, Netherlands

${ }^{\star}$ Corresponding author.

doi: 10.1192/j.eurpsy.2021.309

Introduction: The reward system regulates the processes that motivate people to pursue evolutionary beneficial stimuli. Effective functioning of the reward system can protect against the development of anhedonia. In the daily life, the reward system can be expressed as the dynamic interplay of positive affect (liking), reward anticipation (wanting), and active behavior (engaging). Applying network analysis to daily life experience data allows us to identify such reward dynamics and use them to predict future depressive symptoms.
Objectives: We investigated whether at baseline (i) higher network positive affect in-strength, reflecting how strongly positive affect is influenced by other components and hence the level of anhedonia, and (ii) higher network connectivity, reflecting overall functioning of the reward system, are associated with fewer depressive symptoms on follow-up.

Methods: We used data from 43 participants with mild depressive symptoms from the SMARTSCAN study. The dynamic interplay between momentary positive affect, reward anticipation, and active behavior was assessed with individual vector-autoregressive models and the network analysis. Network positive affect in-strength and connectivity indices were used to predict a six-month depressive symptoms trajectory.

Results: Reward systems networks vary greatly between individuals. On the group level, higher positive affect in-strength (Beta=3.66, $\mathrm{p}=0.05$ ) and network connectivity (Beta=-4.06, $\mathrm{p}=0.03$ ) at baseline were associated with fewer symptoms at follow-up.

Conclusions: Higher influences of reward anticipation and active behavior on positive affect and stronger connections between reward cycle components are associated with fewer future symptoms, suggesting the importance of daily life reward cycle dynamics in depression.

Disclosure: No significant relationships.

Keywords: reward system; reward dynamics; Depression; Network analysis

\section{4}

\section{Potential of antithrombin III as a biomarker of antidepressive effect in major depressive disorder}

R. Song ${ }^{\star}$ and Y. Shi

Department Of Neurology, Affiliated ZhongDa Hospital, School of Medicine, Southeast University, Nanjing, China

${ }^{\star}$ Corresponding author.

doi: $10.1192 /$ j.eurpsy.2021.310

Introduction: Previous study has identified increased antithrombin III (ATIII) in patients with major depressive disorder (MDD), supporting ATIII as a potential biomarker for depression diagnosis. Objectives: This study aimed to reveal the alteration of ATIII after occipital repetitive transcranial magnetic stimulation (rTMS), and illuminate its power to evaluate and predict the curative effects in MDD treatment.

Methods: A total of 90 MDD patients were recruited and further intervened with rTMS in occipital for individualized, standard or sham treatment for five days. Those of 74 patients underwent entire detection, including clinical assessments, blood collection and protein measurement.

Results: After treatment, decreased ATIII were detected in both the individualized and the standard group ( $\mathrm{p}=0.000$ and 0.001 , respectively) instead of the sham one. Especially, the reduction in ATIII in the individualized group was associated with improvements in several neuropsychological assessments. Besides, ATIII at baseline in the standard group and after the individualized rTMS showed high performance to evaluate or predict the response to the 5-day treatment $(\mathrm{AUC}=0.771,95 \% \mathrm{CI}, 0.571-0.971 ; \mathrm{AUC}=0.875,95 \% \mathrm{CI}$, 0.714-1.000, respectively) and the remission in follow-up (AUC=0.736, 95\%CI, 0.529-0.943; AUC=0.828, 95\%CI, 0.6561.000 , respectively). Furthermore, both baseline ATIII and change 